\title{
Validation of a 3D Laser Scanner Structure Sensor to measure upper extremity circumferences: potential clinical implications for lymphoedema rehabilitation.
}

\section{Alessandro de Sire ${ }^{1,2}$, Lorenzo Lippi², Sabrina Pasqua ${ }^{2}$, Monica Pinto3 Francesca Gimigliano ${ }^{4}$, Giovanni Iolascon ${ }^{1}$ Carlo Cisari $^{2}$, Marco Invernizzi $^{2}$}

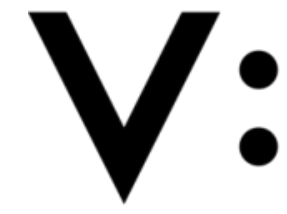

Università degli Studi della Campania Luigi Vanvitelli
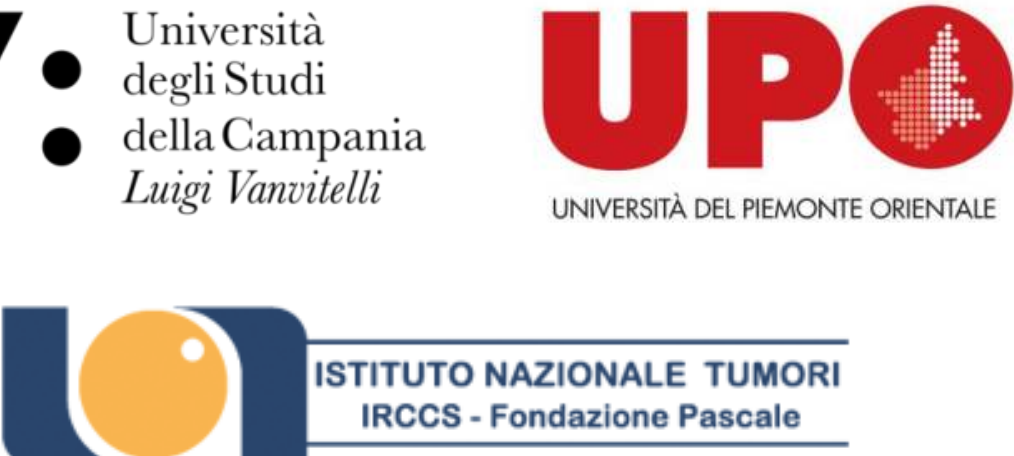

${ }^{1}$ Department of Medical and Surgical Specialties and Dentistry, University of Campania "Luigi Vanvitelli", Naples, Italy. 2 Department of Health Sciences, University of Eastern Piedmont "Amedeo Avogadro" Novara, Italy. ${ }^{3}$ Rehabilitation Medicine Unit, Istituto Nazionale Tumori-IRCCS-Fondazione G. Pascale, Naples, Italy. ${ }^{4}$ Department of Mental and Physical Health and Preventive Medicine, University of Campania "Luigi Vanvitelli", Naples, Italy.

\section{Background}

Upper limb volume assessment is the first step in lymphedema diagnosis and rehabilitative management [1]. Circumferential method (CM), the most commonly used limb volumetric method, showed several limitations in common clinical rehabilitation practice and recently new methods using three-dimensional laser scanning (LS3D) have been proposed for limb volume measurement, even in the worst stages of lymphedema [2-4]. Aim of this study was to verify the reproducibility and reliability of LS3D compared to CM in upper limb volume measurement in healthy young adults and in breast cancer related lymphedema (BCRL) women.

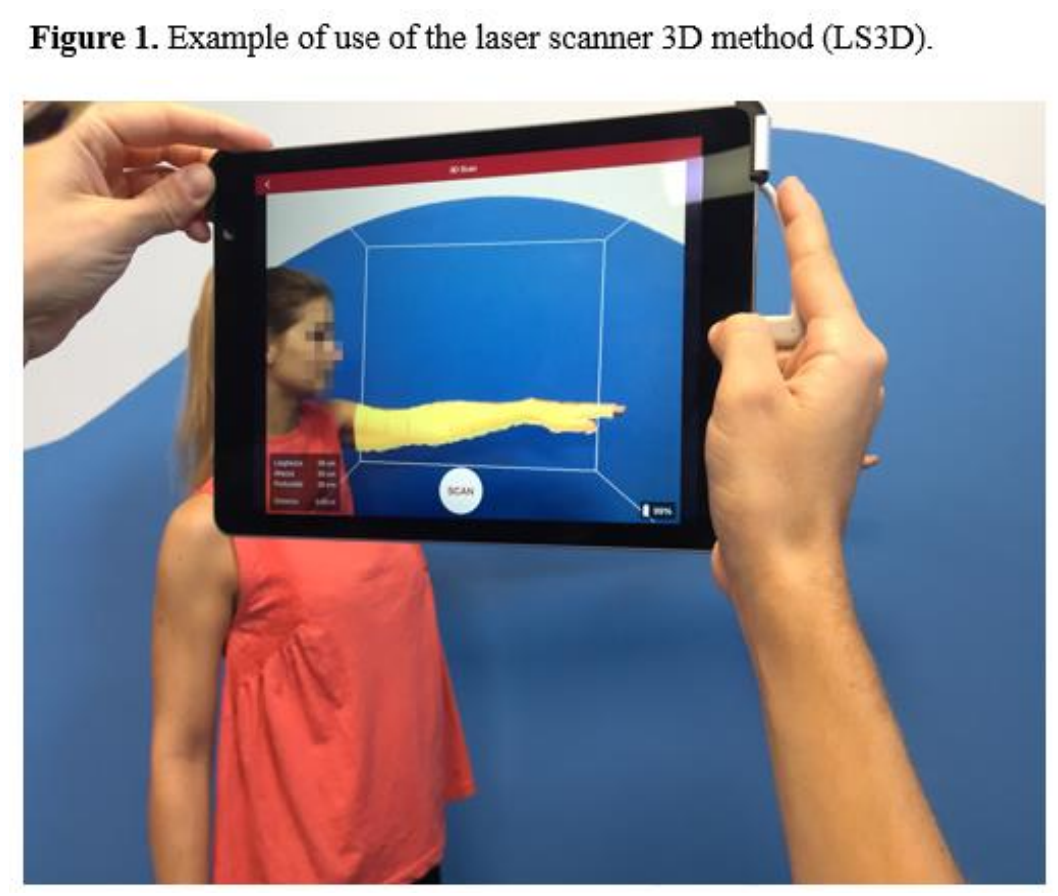

\begin{tabular}{|c|c|c|c|}
\hline \multicolumn{3}{|c|}{ Table 1. Inter-operator analysis of circumferential method } \\
\hline & OPERATOR A & OPERATOR B & MEAN \\
\hline V forearm & $1.04 \pm 0.24$ & $1.04 \pm 0.24$ & $1.04 \pm 0.24$ \\
\hline V arm & $0.92 \pm 0.26$ & $0.92 \pm 0.26$ & $0.92 \pm 0.26$ \\
\hline V tot & $1.96 \pm 0.47$ & $1.96 \pm 0.47$ & $1.96 \pm 0.47$ \\
\hline $\begin{array}{c}\text { Means and standard deviations of volumetric parameters by the } \\
\text { Operator A, Operator B and Inter-operator mean }\left(\mathrm{dm}^{3}\right)\end{array}$ \\
\hline
\end{tabular}

\begin{tabular}{|c|c|c|c|}
\hline \multicolumn{3}{|c|}{ Table 2. Inter-operator analysis of Laser Scanner 3D method } \\
\hline & OPERATOR A & OPERATOR B & MEAN \\
\hline V forearm & $1.02 \pm 0.25$ & $1.02 \pm 0.25$ & $1.02 \pm 0.25$ \\
\hline V arm & $0.89 \pm 0.26$ & $0.90 \pm 0.26$ & $0.89 \pm 0.26$ \\
\hline V tot & $1.91 \pm 0.48$ & $1.91 \pm 0.49$ & $1.91 \pm 0.49$ \\
\hline $\begin{array}{c}\text { Means and standard deviations of volumetric parameters by the } \\
\left.\text { Operator A, Operator B and Inter-operator mean (dm }{ }^{3}\right)\end{array}$ \\
\hline
\end{tabular}

Figure 2. Correlation between laser scan 3D and circumferential method.

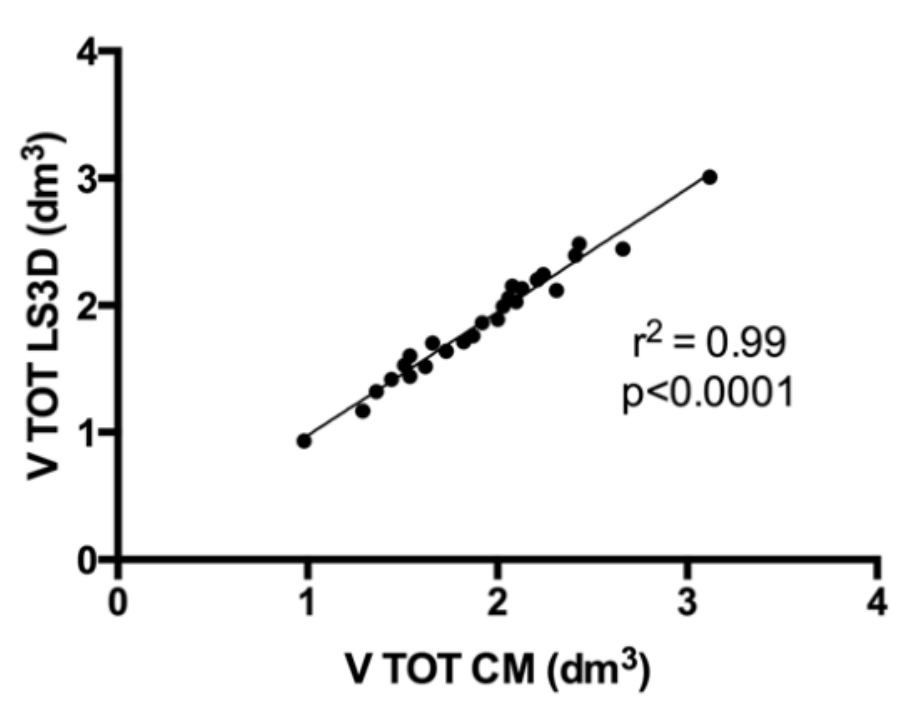

Methods

Healthy young adults, without diseases altering upper limb volume and BCRL patients were enrolled in the Physical and Rehabilitative Medicine Service, University Hospital "Maggiore della Carità", Novara, Italy. Two raters performed both LS3D (Figure 1) and centimetric upper limb volume evaluation twice in each subject. Each procedure duration was measured.

\section{Results}

Thirty healthy young adults ( 14 male and 16 female), mean aged $27.6 \pm 9.8$ years with a mean BMI of $22.7 \pm 2.9 \mathrm{~kg} / \mathrm{m} 2$ were enrolled. Both LS3D and CM showed a significant inter-operator (Tables 1 and 2) and intra-operator correlation in upper limb volume measurement $\left(r^{2}=0.99 ; p<0.0001\right)$. Moreover LS3D showed a strong correlation with $\mathrm{CM}\left(r^{2}=0.99 ; p<0.0001\right)$ (Figure 2).

LS3D was significantly quicker in upper limb volume measurement than CM (202 \pm 27 sec. vs $293 \pm 17 \mathrm{sec} ; \mathrm{p}<0.0001)$. In the preliminary analysis performed on 4 women affected by BCRL, LS3D showed the same inter and intra-operator correlation than in healthy subjects $\left(r^{2}=0.99 ; p<0.0001\right)$.

\section{Conclusions}

LS3D Structure Sensor is a highly reproducible, reliable and easy to use method to evaluate upper limb volume in healthy subjects. These results combined with the preliminary ones obtained in BCRL patients suggest its potential use in lymphedema common clinical rehabilitation practice.

Bibliografia

1. International Society of Lymphology. The diagnosis and treatment of peripheral lymphedema. Consensus document of the International Society of Lymphology. Lymphology 2003; 36(2): 84-91.

2. Mayrovitz M, Sims N, Hill C, Hernandez T, Greenshner A, Diep H. Hand volume estimates based on a geometric algorithm in comparison to water displacement. Lymphology. 2006; 39: 95-103.

3. Harrison JA, Nixon MA, Fright WR, Snape L. Use of hand-held laser scanning in the assessment of facial swelling: a preliminary study. Br J Oral Maxillofac Surg. 2004 Feb;42(1):8-17.

4. Cau N, Galli M, Cimolin V, Aranci M, Caraceni A, Balzarini A. Comparative study between circumferential method and laser scanner 3D method for the evaluation of arm volume in healthy subjects. J Vasc Surg Venous Lymphat Disord. 2016 Jan;4(1):64-72. 